\title{
Background Synaptic Activity Is Sparse in Neocortex
}

\author{
Jack Waters and Fritjof Helmchen \\ Abteilung Zellphysiologie, Max-Planck-Institut für Medizinische Forschung, 69120 Heidelberg, Germany
}

\begin{abstract}
Neurons are continually exposed to background synaptic activity in vivo. This is thought to influence neural information processing, but background levels of excitation and inhibition remain controversial. Here we show, using whole-cell recordings in anesthetized rats, that spontaneous depolarizations ("Up states") in neocortical pyramidal neurons are driven by sparse, mostly excitatory synaptic activity (less than five inputs per millisecond; $\sim 10 \%$ inhibitory). The mean synaptic conductance change is small ( $<10 \mathrm{nS}$ at the soma) and opposed by anomalous rectification, resulting in a net increase in input resistance during Up states. These conditions enhance the effectiveness of each synapse at depolarized potentials. Hence, neocortical networks are relatively quiet at rest, and the effect of synaptic background is weaker than previously thought.
\end{abstract}

Key words: pyramidal cell; summation; patch clamp; dendrite; synaptic integration; dendrite

\section{Introduction}

The principal task of every neuron is to interpret the patterns of synaptic input arriving in the dendritic tree and generate appropriate action potential (AP) output, a process termed synaptic integration (Magee, 2000). In the CNS, unitary EPSPs are small, so many inputs must summate to reach AP threshold (Häusser et al., 2000; Magee, 2000). Spontaneous activity within surrounding neural networks makes synaptic integration in vivo even more difficult by producing a noisy background of ongoing synaptic input (Bernander et al., 1991; Destexhe and Paré, 1999; Steriade, 2001; Destexhe et al., 2003). Background activity causes membrane depolarization, often in the form of periodically occurring "Up states" (Steriade et al., 1993, 2001; Wilson and Kawaguchi, 1996), reducing the driving force for EPSCs. In addition, synaptic input can shunt the membrane, reducing input resistance and EPSP amplitudes (Bernander et al., 1991), and this has led to the suggestion that Up states are "high-conductance states" (Destexhe et al., 2003)

Neurons have developed properties to diminish or overcome this shunting problem. These properties include the spatial distribution of synapses throughout the dendritic tree, which compartmentalizes conductance changes (Polsky et al., 2004; Williams, 2004), and the expression of voltage-gated ion channels, which can counteract synaptically induced conductance changes by increasing input resistance with depolarization (anomalous rectification) (Katz, 1949; Wilson, 1992).

Although shunting, synaptic compartmentalization and rec-

Received May 21, 2006; revised June 29, 2006; accepted July 3, 2006.

We thank Bert Sakmann for his encouragement and generous support. We are especially grateful to Arnd Roth for discussions and help with simulations. The L2/3 pyramidal cell morphology was kindly provided by Dirk Feldmeyer We thank Jason Kerr, Arnd Roth, and Bert Sakmann for critically reading this manuscript and Marlies Kaiser for expert technical assistance.

Correspondence should be addressed to Jack Waters, Department of Physiology, Feinberg School of Medicine, Northwestern University, 303 East Chicago Avenue, Chicago, IL 60611. E-mail: jackwaters@northwestern.edu.

F. Helmchen's present address: Department of Neurophysiology, Brain Research Institute, University of Zurich, Winterthurerstrasse 190, 8057 Zurich, Switzerland.

DOI:10.1523/JNEUROSCI.2152-06.2006

Copyright $\odot 2006$ Society for Neuroscience $\quad$ 0270-6474/06/268267-11\$15.00/0 tification are well known phenomena, how they interact to determine net input resistance and EPSP summation in the intact brain is unclear. In part, this results from a lack of information about the numbers of background synapses active in vivo. Here, we examine spontaneous background synaptic activity in the neocortex of the anesthetized rat. We show that somatic input resistance increases during Up states and demonstrate that this increase is attributable to anomalous rectification in these neurons. We then estimate the mean numbers of active excitatory and inhibitory synapses. We find that far fewer synapses are spontaneously active in cortical networks than previously thought and that the majority of these active synapses are excitatory.

\section{Materials and Methods}

\section{In vivo electrophysiology}

All experimental procedures were performed in accordance with the animal welfare guidelines of the Max Planck Society. Wistar rats [postnatal day 27 (P27) to P32] were anesthetized with urethane (1-2 g/kg). Depth of anesthesia was sufficient to eliminate pinch withdrawal, corneal reflex, and vibrissal movements. A small $(2 \times 2 \mathrm{~mm})$ craniotomy was opened over barrel cortex, and the dura was removed (coordinates for center of craniotomy: $2 \mathrm{~mm}$ anterior to bregma, $5-6 \mathrm{~mm}$ lateral). The craniotomy was covered with agar (1-1.5\%, type III-A; Sigma, St. Louis, MO) in the following solution (in $\mathrm{mM}$ ): $135 \mathrm{NaCl}, 5.4 \mathrm{KCl}, 1 \mathrm{MgCl}_{2}, 1.8 \mathrm{CaCl}_{2}$, and 5 HEPES. A glass coverslip was positioned over the agar to reduce brain pulsation.

Whole-cell patch-clamp recordings were obtained using a "blind" technique as described previously (Margrie et al., 2002). Recording pipettes $(4-6 \mathrm{M} \Omega$ ) were filled with an intracellular solution containing the following: $135 \mathrm{~mm}$ K-gluconate, $4 \mathrm{~mm} \mathrm{KCl,} 10 \mathrm{~mm} \mathrm{HEPES}, 10 \mathrm{~mm} \mathrm{Na}_{2}$ phosphocreatine, $4 \mathrm{~mm}$ Mg-ATP, $0.3 \mathrm{~mm} \mathrm{Na-GTP,} 200 \mu \mathrm{m}$ Oregon Green BAPTA 488-1, $20 \mu \mathrm{m}$ Alexa 594, 0.2\% (w/v) biocytin, pH 7.2, 291-293 mOsm. Positive pressure (200-400 mbar) was applied to the pipette as it was inserted through the agar and the pial surface of the cortex. The positive pressure was reduced to 25-30 mbar when the tip was at approximately the upper limit of L2/3. The pipette was then advanced in $2 \mu \mathrm{m}$ steps. Voltage pulses were applied to the pipette (10-20 $\mathrm{mV} ; 30 \mathrm{~ms} ; 10 \mathrm{~Hz}$ ), and the current response monitored. Positive pressure was relieved when the series resistance of the electrode abruptly increased after a $2 \mu \mathrm{m}$ step, indicating that the tip of the pipette may have 
been pushed against a neuronal plasma membrane. Gentle suction (up to 100 mbar) was applied where necessary to obtain a gigaohm seal. Initial access resistance in the whole-cell configuration was typically 30-60 $\mathrm{M} \Omega$. All recordings were obtained using an Axoclamp 2B amplifier (Molecular Devices, Union City, CA). L2/3 pyramidal neurons were identified as in our previous studies using two-photon microscopy and histological reconstructions (Waters et al., 2003; Waters and Helmchen, 2004). Somatic depths were $150-500 \mu \mathrm{m}$ below the pia.

Sharp microelectrode recordings were obtained using 100-120 M $\Omega$ microelectrodes filled with $2.5 \mathrm{~m}$ potassium acetate. Microelectrodes were advanced in $2 \mu \mathrm{m}$ steps. Typically, their resistance decreased during passage through the tissue to $\sim 70-90 \mathrm{M} \Omega$ before a recording was obtained. After penetration, the neurons were found to be depolarized (to around $-40 \mathrm{mV}$ ) and current was injected to hold the membrane potential at around $-70 \mathrm{mV}$. In the subsequent 5-10 min, the membrane potential stabilized and the holding current could be reduced (to zero). The neuron was allowed to recover its membrane potential before data collection; no holding current was used during data collection.

\section{Input resistance measurements and data analysis}

Brief pulse protocol. Brief current pulses ( $-300 \mathrm{pA}$ for $80 \mathrm{~ms}$ ) were injected through the recording pipette at $200 \mathrm{~ms}$ intervals, producing hyperpolarizing voltage deflections of $\sim 5-10 \mathrm{mV}$. This allowed sufficient time for the membrane potential to relax to a steady-state value before measurement, both during and after the pulse. Access resistance was monitored every $2-10 \mathrm{~s}$, rebalancing the bridge if necessary. Data were discarded if access resistance changed by $>10 \mathrm{M} \Omega$ over a 1 min period or $>20 \mathrm{M} \Omega$ over the entire data collection period (typically $5 \mathrm{~min}$ ).

We sorted the resulting voltage responses according to whether they occurred during Up or Down states, manually defining Up and Down state thresholds $\sim 3-5$ and $10-15 \mathrm{mV}$ more depolarized than the Down state membrane potential, respectively (see Fig. $1 C$ ). We then calculated the mean membrane potential during two $10 \mathrm{~ms}$ time windows; one 1-11 $\mathrm{ms}$ before the start of the pulse, and the other 30-40 ms after termination of the pulse. Pulses were defined as occurring during Down states when the membrane potential was below the Down state threshold in both time windows (before and after the pulse). Similarly, pulses were defined as occurring during Up states if the membrane potential was above the Up state threshold in both windows. Pulses not meeting either of these criteria, such as those during which a state transition occurred, were sorted into a third group, termed "Discards." Input resistance was derived for each group according to Ohm's law, by dividing the mean voltage deflection during the second half of the current pulse by the current amplitude $(-300 \mathrm{pA})$. Decay time constants were derived from the mean voltage responses by fitting a single-exponential curve to the decay of the voltage trace, starting $1 \mathrm{~ms}$ after the end of the current pulse.

Constant current injection protocol. Constant holding currents of up to $\pm 1.6 \mathrm{nA}$ amplitude were injected at $200 \mathrm{pA}$ intervals. The order in which we imposed different currents was semirandomized to avoid systematic artifacts resulting from changes in cellular properties during prolonged depolarization or hyperpolarization. We recorded the membrane potential for 1 min during the injection of each current, starting 10-30 s after the start of the current injection to ensure steady-state conditions. For each period, a membrane potential histogram was calculated as a frequency distribution of the membrane potential, using a bin width of 0.1 $\mathrm{mV}$. The absolute frequency was divided by the total number of points to give the probability for each membrane potential bin. Cumulative frequency histograms were calculated as the integrals of the membrane potential histograms.

All data are presented as mean \pm SEM. Except where otherwise stated, all statistical analyses were performed using the two-tailed Wilcoxon matched-pairs signed-rank test in GraphPad InStat, version 3.05 (GraphPad, San Diego, CA).

\section{Reversal potential measurements}

The potential at which Up states reversed was measured using the following intracellular solution: $135 \mathrm{~mm}$ Cs-gluconate, $4 \mathrm{~mm} \mathrm{CsCl,} 10 \mathrm{~mm}$ HEPES, $10 \mathrm{~mm} \mathrm{Na}$-phosphocreatine, $0.3 \mathrm{~mm} \mathrm{Na}-\mathrm{GTP}, 0.2 \mathrm{~mm}$ Oregon
Green BAPTA 488-1, 0.02 mm Alexa 594, 0.2\% (w/v) biocytin, $10 \mathrm{~mm}$ (2,6-dimethylphenyl)carbamoylmethyl-triethyl-azanium (QX-314). Recordings at various membrane potentials were made using discontinuous current clamp (sampling frequency, 3-4.5 kHz). Distinguishing between Up and Down states in the whole-cell recording became difficult where the Up state amplitude was small, around the Up state reversal potential. We therefore simultaneously monitored Up and Down states in the electrocorticogram (ECoG) as described previously (Waters and Helmchen, 2004) and used the ECoG signal to determine when transitions between Up and Down states occurred. We defined a transition between states as occurring each time the smoothed ECoG signal crossed a threshold equal to the mean of the ECoG signal. This objective approach accurately identified many (although not all) Up and Down state transitions in the whole-cell recordings. The Up and Down state membrane potentials were then calculated as the mean voltages during each state. The membrane potential difference between Up and Down states $(\Delta V)$ was then plotted against the mean Down state membrane potential and fit with a straight line. The reversal potential of the Up state was determined as the intersection of the fit with the abscissa.

The ratio of mean inhibition to mean excitation $\left(\left\langle g_{i}\right\rangle /\left\langle g_{e}\right\rangle\right)$ relates to the reversal potential $\left(E_{\text {rev }}\right)$ as follows: $\left\langle g_{\mathrm{i}}\right\rangle /\left\langle g_{\mathrm{e}}\right\rangle=\left(E_{\text {rev }}-E_{\mathrm{e}}\right) /\left(E_{\mathrm{i}}-E_{\text {rev }}\right)$, where $E_{\mathrm{e}}$ and $E_{\mathrm{i}}$ are the reversal potentials of excitatory and inhibitory synapses, respectively. Excitatory synaptic currents reverse at $\sim 0 \mathrm{mV}$. From our intracellular and extracellular solutions, we estimate (from the Nernst equation) $E_{\mathrm{i}}$ to be approximately $-95 \mathrm{mV}$. Hence, a $\left\langle g_{\mathrm{i}}\right\rangle /\left\langle g_{\mathrm{e}}\right\rangle$ ratio of 0.1 would produce an Up state reversal potential of $-9 \mathrm{mV}$.

Recordings were obtained with a $\mathrm{Cs}^{+}$-based intracellular solution and QX-314 to maximize voltage control and suppress spiking. Nonetheless, broad spikes were sometimes observed near threshold. We discarded traces containing spikes, leading to the absence of $V-I$ data points between approximately -30 and $-5 \mathrm{mV}$.

\section{Mathematical description of anomalous rectification}

Anomalous rectification in cortical pyramidal neurons has been reported by many authors (Connors et al., 1982; Stafstrom et al., 1982, 1985; Spain et al., 1987; Sutor and Zieglgänsberger, 1987). We used a novel mathematical description to quantify rectification in pyramidal neurons. The $V-I$ relationship of a passive membrane is a simple linear function $\Delta V=$ $R_{N, 0} \Delta I$ with a voltage-independent input resistance $R_{N, 0}$. Here, we included rectification by introducing an additional quadratic term with coefficient $c_{\mathrm{AR}}$ :

$$
\Delta V=R_{N, 0} \Delta I+c_{\mathrm{AR}} \Delta I^{2} .
$$

This simple phenomenological description fit our experimentally determined $V-I$ curves well. To incorporate this rectification model into the NEURON simulation environment (see below), we needed to derive an equation that describes the leak current as a function of voltage deviation from the resting membrane potential. This equation is the inverse function of Equation 1:

$$
\Delta I(V)=\frac{1}{2 c_{\mathrm{AR}}}\left(-R_{N, 0}+\sqrt{R_{N, 0}^{2}+4 c_{\mathrm{AR}}\left(V-V_{\text {rest }}\right)}\right),
$$

with depolarizing voltage deflections producing positive (outward) currents. An analytical expression for the voltage dependence of input resistance can then be obtained by taking the derivative of Equation 1, which by definition equals the input resistance:

$$
\frac{\partial V(I)}{\partial I}=R_{N}(I)=2 c_{\mathrm{AR}} \Delta I+R_{N, 0}
$$

Insertion of Equation 2 into Equation 3 results in a simple expression for the voltage dependence of input resistance, which allows us to calculate the expected input resistance at any membrane potential (e.g., the Up state level) as follows:

$$
R_{\mathrm{Up}}, I V=\sqrt{R_{N, 0}^{2}+4 c_{\mathrm{AR}}\left(V_{\mathrm{Up}}-V_{\mathrm{rest}}\right)} .
$$


Input resistance measurements using current pulses yield values slightly different from the value at the initial membrane potential, depending on the direction and amplitude of the resulting voltage deflections (often termed "apparent" input resistances). When comparing input resistances derived from current pulses and from the slopes of $V-I$ relationships, we accounted for the current-induced voltage change by extracting expected input resistances (from $V-I$ relationships) at the midpoint potential (i.e., the potential halfway between the resting potential and that obtained during the current pulse).

\section{Numerical models}

The goal of modeling was to obtain an estimate for the number of active synapses during spontaneous activity in vivo. To this end, we reconstructed Up states using simple numerical models constrained by our experimental results. Rather than explicitly including active conductances, we simply simulated a membrane with anomalous rectification as described above. Numerical simulations were performed in the NEURON simulation environment (NEURON 5.7 for Windows) (Hines and Carnevale, 1997). The standard passive leak current was replaced by an anomalously rectifying distributed membrane mechanism including the additional parameter for anomalous rectification $c_{\mathrm{AR}}$.

First, we constructed a point conductance model of an isopotential neuron, which was equipped with an anomalously rectifying membrane and which received both excitatory and inhibitory synaptic input. In accordance with our experimental results, input resistance was adjusted to $30 \mathrm{M} \Omega$, membrane time constant to $9 \mathrm{~ms}$, and the anomalous rectification parameter to $c_{\mathrm{AR}}=18 \mathrm{M} \Omega / \mathrm{nA}$. Excitatory synaptic conductance changes were simulated using a reversal potential of $0 \mathrm{mV}$, a peak amplitude of $1 \mathrm{nS}$, and a double-exponential time course with a rise time constant of $0.2 \mathrm{~ms}$ and a decay time constant of $1.7 \mathrm{~ms}$ (Häusser and Roth, 1997). Inhibitory inputs were simulated using a reversal potential of $-75 \mathrm{mV}$, a $0.5 \mathrm{nS}$ peak conductance change, and rise and decay time constants of 1 and $10 \mathrm{~ms}$, respectively (Ali et al., 2001). The mean total synaptic conductance change was adjusted to $8 \mathrm{nS}( \pm 3 \%)$. Synaptic input was modeled using Poisson-distributed presynaptic spike trains. Note that for the point conductance model, many excitatory (or inhibitory) inputs are combined into one input. Because spike intervals were exponentially distributed, the mean spike rate of the merged input is equivalent to $n$-times the mean rate for $n$ synapses, each activated at the same rate. To compare our results with high-conductance models of Up states, we also constructed Up states under the assumption of an $80 \%$ drop in input resistance without anomalous rectification. In this case, a $>10$-fold higher total mean synaptic conductance change of $138 \mathrm{nS}$ $( \pm 3 \%)$ had to be used. Under these conditions, to keep the membrane potential depolarization within the experimental bounds, the ratio of inhibition to excitation had to be 3-4.

For a more realistic simulation, we used a compartmental model of a L2/3 pyramidal neuron based on a detailed morphological reconstruction kindly provided by D. Feldmeyer and A. Roth (Lübke et al., 2003). A specific membrane resistance of $4350 \Omega \mathrm{cm}^{2}$ and an axial resistance of $150 \Omega \mathrm{cm}$ were used. Specific membrane capacitance was $1 \mu \mathrm{F} / \mathrm{cm}^{2}$ in the soma and $2 \mu \mathrm{F} / \mathrm{cm}^{2}$ in dendrites (to account for dendritic spines). Anomalous rectification was adjusted to yield $c_{\mathrm{AR}}=18 \mathrm{M} \Omega / \mathrm{nA}$. A total of 10,000 excitatory and 2000 inhibitory synapses (DeFelipe and Fariñas, 1992) (with properties as in the point conductance model) was uniformly distributed throughout the dendrites. To determine input resistances in this model, we applied the same brief pulse protocol as in our experiments. In some experiments, additional synaptic input mimicking the afferent synapses from L4 spiny stellate cells to L2/3 neurons was added in the basal dendritic tree ( 25 synchronized synaptic inputs at a mean distance $68 \mu \mathrm{m}$ from the soma) (Feldmeyer et al., 2002). The summation of synchronous input was also evaluated during both Down and Up states by increasing the number of synapses on the basal dendrites (maximum number of 125 , with a mean distance from the soma of $77.6 \mu \mathrm{m})$.

\section{Results}

Input resistance increases during $\mathrm{Up}$ states in $\mathrm{L} 2 / 3$ neurons

Whole-cell recordings were obtained from L2/3 neocortical pyramidal neurons in young adult rats under urethane anesthesia. Somatic voltage recordings revealed a stereotypical pattern of spontaneous $10-20 \mathrm{mV}$ membrane potential $\left(V_{\mathrm{m}}\right)$ fluctuations between a hyperpolarized Down state and a depolarized Up state (Fig. 1A). Up states mostly remained subthreshold with spontaneous APs occurring infrequently $\left(0.089 \pm 0.024 \mathrm{APs} \mathrm{s}^{-1}\right.$; range, $0-0.4$ APs s $^{-1} ; n=29$ cells), consistent with previous reports (Margrie et al., 2002; Brecht et al., 2003).

To compare somatic input resistance $\left(R_{N}\right)$ during Up and Down states, we used three approaches. First, brief hyperpolarizing current pulses were delivered at regular intervals (Fig. $1 B$ ). During Down states, each current pulse produced a similar hyperpolarizing response. In contrast, current injections during Up states resulted in voltage responses with a wide range of amplitudes, some pulses producing larger and others smaller hyperpolarizations compared with the Down states (Fig. $1 B$ ). For each neuron, several hundred voltage responses were collected and then sorted into three groups, according to whether they occurred during Down states, Up states, or during state transitions (the latter termed the "Discards" group) (Fig. 1C). Surprisingly, the mean input resistance was higher during Up states than during Down states in every neuron examined (Fig. 1C,D) $(29.2 \pm$ 2.0, 31.6 \pm 2.2 , and 36.6 $\pm 2.3 \mathrm{M} \Omega$ for Down, Discards, and Up groups, respectively; $n=16$ neurons; $p<0.0001$ for Up vs Down).

Current-evoked voltage responses could be subject to errors caused by changes in access resistance or incorrect bridge balance. We therefore also used a second approach, analyzing the decay of the hyperpolarizing voltage deflections after the end of the current pulse. The time constant for the tail of this decay is insensitive to access resistance, but directly relates to input resistance (Rall, 1969; Koch, 1999). Single exponential curves were fit to the decay of the mean voltage deflections for all three groups (Fig. 1C). The time constant was longer during Up states than during Down states in all neurons examined (Fig. 1E) (mean values, $9.0 \pm 0.6,10.6 \pm 0.6$, and $12.8 \pm 1.1 \mathrm{~ms}$ for Down, Discards, and Up groups, respectively; $n=16$ neurons; values for Up and Down states were significantly different, $p<0.0001$ ). These results are consistent with an increased mean input resistance during Up states.

A third method for measuring input resistance changes during spontaneous activity is to inject constant current and analyze the resulting steady-state membrane potential changes during Up and Down states. If input resistance differs between Up and Down states, the steady-state membrane potentials of the two states will change by different amounts (Paré et al., 1998). In L2/3 neurons, we observed a significant $R_{N}$ increase during Up states using this approach (Fig. 2) (37.9 \pm 7.0 and $42.0 \pm 8.2 \mathrm{M} \Omega$ for Down and Up states, respectively; $p=0.02$ ). Hence, using three complementary techniques, we consistently found that $R_{N}$ increases by $10-30 \%$ during Up states. Similar results were obtained in three additional dendritic recordings and in two recordings of fast-spiking neurons. Data are summarized in Table 1.

Our finding that $R_{N}$ increases during Up states contrasts with several previous studies reporting a decrease of up to $80 \%$ in cortical pyramidal neurons (Paré et al., 1998; Steriade et al., 2001; Destexhe et al., 2003; Léger et al., 2005). To examine whether different techniques (whole-cell recording vs sharp microelec- 
trode impalements) might have produced such different results, we repeated measurements with sharp microelectrodes. In four microelectrode recordings, we found no significant difference between Up and Down state input resistances using all three approaches described above (Table 1; supplemental Fig. 1, available at www. jneurosci.org as supplemental material). We conclude that recording technique alone cannot account for the difference between our results and those of previous studies.

\section{Voltage dependence of Up and Down state durations}

Up states are driven by volleys of synaptic activity (Cowan and Wilson, 1994; Petersen et al., 2003). How can $R_{N}$ increase during such active periods, which are associated with the opening of synaptic conductances?

A clue came from our constant current injection experiments, in which the proportion of time spent in Up and Down states changed with membrane potential. This voltage dependence was evident in the raw data (Fig. $3 a$ ) and in cumulative membrane potential frequency histograms, in which the point of inflection shifted toward lower values with depolarization (Fig. 3b). Hence, when depolarized, neurons spent more time in the Up state and, when hyperpolarized, more time in the Down state. This effect was observed in all five neurons examined. To quantify the voltage dependence, we plotted the ratio of time spent in Up and Down states as a function of the membrane potential shift caused by constant current injection. The slope of a linear fit to the pooled data indicated that the fraction of time spent in the Up state changed by $1.7 \pm 0.9 \%$ per millivolt of depolarization or hyperpolarization (Fig. 3c).

A similar voltage dependence of Up and Down state durations has been reported for cortical neurons (Cowan and Wilson, 1994) and for neostriatal spiny projection neurons, in which it was attributed to the opening of intrinsic potassium conductances with hyperpolarization, which decreases input resistance. It has been suggested that this increases the number of excitatory synapses that must be activated to initiate an Up state and thereby reduces Up state duration (Wilson and Kawaguchi, 1996). Intrinsic conductances operating in the subthreshold voltage regimen may, likewise, influence Up and Down state durations and input resistance in $\mathrm{L} 2 / 3$ pyramidal neurons. We therefore more closely investigated the intrinsic subthreshold membrane properties in L2/3 pyramidal neurons.
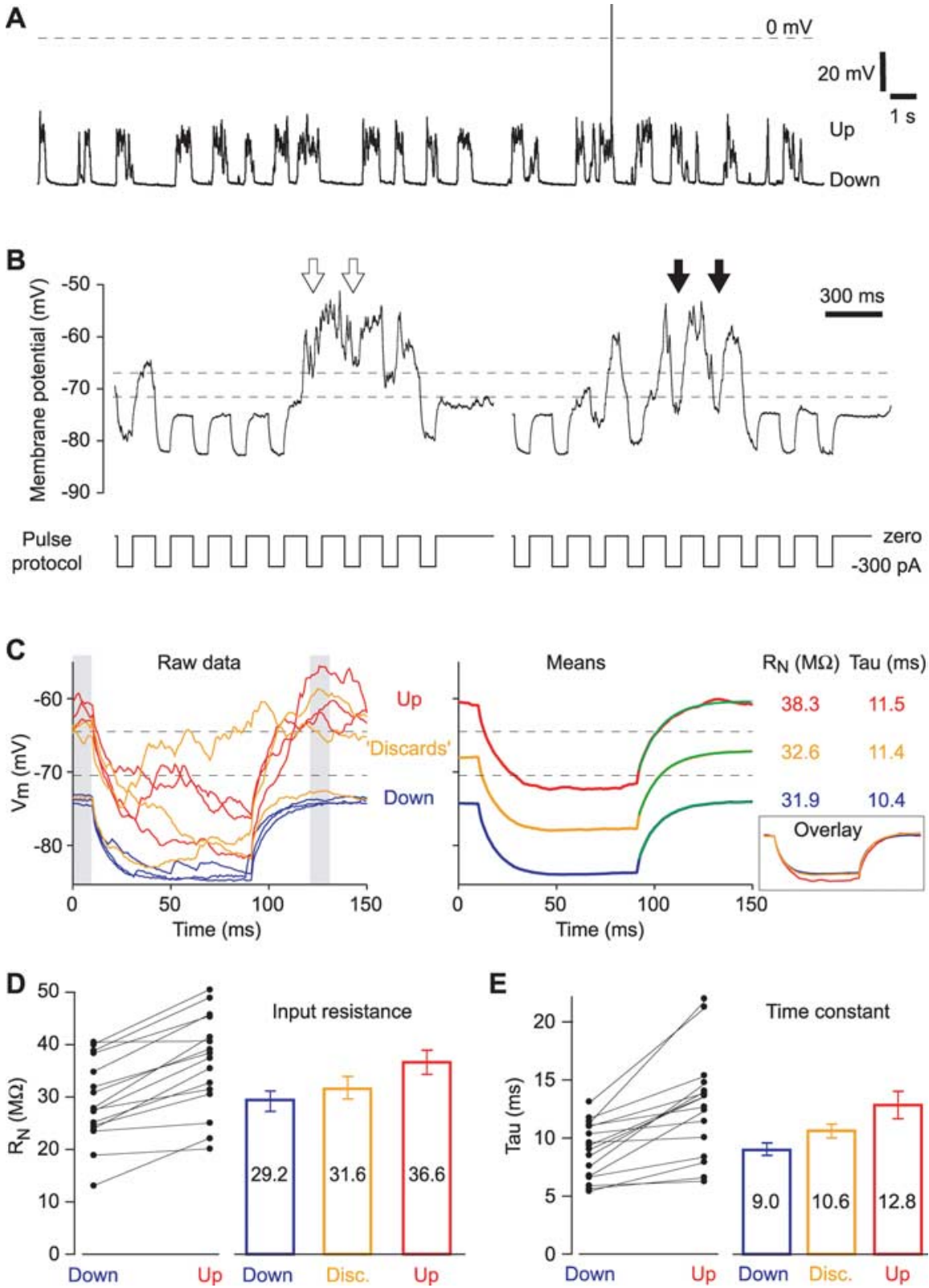

Figure 1. Input resistance during Up and Down states. $\boldsymbol{A}$, Spontaneous membrane potential fluctuations (Up and Down states) recorded from a $\mathrm{L} 2 / 3$ pyramidal neuron in vivo. $\boldsymbol{B}$, Two examples of membrane potential recordings during application of brief current pulses to measure input resistance. The dashed lines are voltage thresholds for detection of Up and Down states. Eighty millisecond pulses of $-300 \mathrm{pA}$ were delivered at $5 \mathrm{~Hz}$. The arrows mark examples of pulses during Up states that produced voltage deflections smaller (open arrows) or larger (filled arrows) than deflections during Down states. $\boldsymbol{C}$, Left, Nine example traces (each showing 1 voltage response) from a continuous 2 s sweep of data. The dashed lines are Up and Down state thresholds ( -64.5 and $-70.5 \mathrm{mV}$, respectively). The gray shaded regions denote the two time windows for Up/Down state classification. Each trace is colored according to the group into which it was classified (Up state, red; Down state, blue; discards, yellow). Right, Average voltage deflections for this neuron for pulses occurring during Up states (50 pulses), Down states (157 pulses), and discards (243 pulses). These average voltages were used to calculate input resistances (right). Single exponential curves were fit to the decay of the voltage deflections after each current pulse (green). Time constants are given on the right. Inset, Overlay of average traces. $\boldsymbol{D}$, Comparison of input resistance during Up and Down states for 16 neurons. Left, Individual experiments. Right, Mean \pm SEM. $\boldsymbol{E}$, Comparison of time constants during Up and Down states for 16 neurons. Left, Individual experiments. Right, Mean \pm SEM.

\section{Anomalous rectification in L2/3 pyramidal neurons}

Input resistance is defined as the slope of the voltage-current $(V-I)$ relationship. Hence any voltage-dependent changes will appear as rectification in the $V-I$ relationship (Koch, 1999). To examine the role of voltage-dependent currents, we therefore obtained steady-state voltage-current $(V-I)$ relationships from 10 neurons, all of which displayed anomalous (inward) rectifica- 

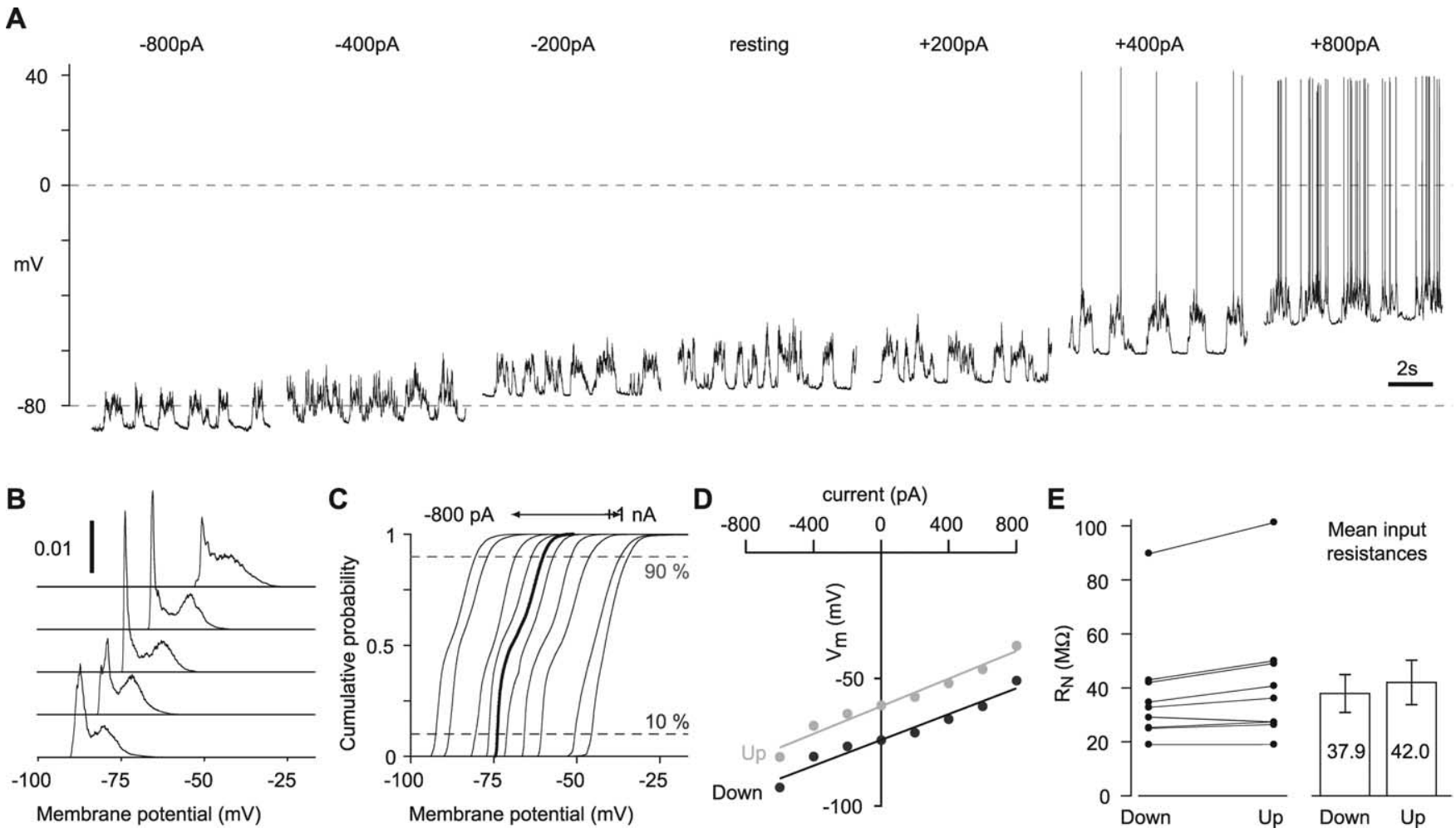

Mean input resistances

Figure 2. Parallel shift of Up and Down states with constant holding currents. $A$, Membrane potential recordings from a single $L 2 / 3$ neuron during hyperpolarizing and depolarizing constant current injections from -0.8 to $+0.8 \mathrm{nA}$ (indicated above each trace). $\boldsymbol{B}$, Membrane potential histograms for the neuron shown in $\boldsymbol{A}$. Bin width, $0.1 \mathrm{mV}$. For clarity, only histograms for current injections of $-0.8,-0.4$, zero, +0.4 , and $+0.8 \mathrm{nA}$ are shown. Histogram integrals are normalized to 1 . C, Cumulative membrane potential histograms for the same neuron. Ten current injections from $-800 \mathrm{pA}$ to $+1 \mathrm{nA}$ (in $200 \mathrm{pA}$ steps) are shown. The thicker line denotes the histogram at resting membrane potential. The dashed horizontal lines indicate the 10 th and 90 th percentiles. $\boldsymbol{D}$, $\mathrm{V}-$ / plots for 10th (black markers) and 90th (gray markers) percentiles, derived from the cumulative membrane potential histograms in $\boldsymbol{C}$. The slopes of the fitted lines give input resistances in Down (black line) and Up (gray line) state, respectively. $\boldsymbol{E}$, Comparison of input resistances measured by constant current injection during Up and Down states for nine neurons. Left, Individual experiments. Right, Mean \pm SEM.

Table 1. Electrophysiological properties of neocortical L2/3 neurons in vivo

\begin{tabular}{|c|c|c|c|c|c|}
\hline & $\begin{array}{l}\text { Pyramidal cell somatic } \\
\text { recording }(n=16)\end{array}$ & $\begin{array}{l}\text { Pyramidal cell } \\
\text { dendritic recording }^{a} \\
(n=3)\end{array}$ & $\begin{array}{l}\text { FS interneuron } \\
(n=2)\end{array}$ & $\begin{array}{l}\text { Pyramidal cell } \\
\text { microelectrode } \\
\text { recording }(n=3)\end{array}$ & $\begin{array}{l}\text { Pyramidal cell with } \\
\text { QX-314 }(n=4)\end{array}$ \\
\hline Resting $V_{m^{\prime}}$ Down (mV) & $-73.9 \pm 1.4$ & $-78.7 \pm 2.2$ & $-76.1 \pm 6.4$ & $-75.3 \pm 3.4$ & $-63.1 \pm 3.3$ \\
\hline Resting $V_{m^{\prime}}{ }^{\prime}$ Up (mV) & $-58.9 \pm 1.2$ & $-66.2 \pm 1.2$ & $-56.8 \pm 5.2$ & $-61.2 \pm 4.8$ & $-47.9 \pm 2.7$ \\
\hline$\Delta V$ (Up-Down) (mV) & $15.0 \pm 0.9$ & $12.6 \pm 1.8$ & $19.4 \pm 1.2$ & $14.1 \pm 2.1$ & $15.1 \pm 3.4$ \\
\hline$V_{\mathrm{m}} \mathrm{SD}, \mathrm{Up}(\mathrm{mV})$ & $2.5 \pm 0.1$ & $3.2 \pm 0.4$ & $3.4 \pm 0.3$ & $3.2 \pm 0.3$ & \\
\hline Mean dwell time, Down (ms) & $347 \pm 32$ & & & $343 \pm 24(n=4)$ & \\
\hline Mean dwell time, Up (ms) & $457 \pm 57$ & & & $224 \pm 26(n=4)$ & \\
\hline AP threshold (mV) & $-36.4 \pm 1.0$ & $-44.3 \pm 2.7$ & $-34.1 \pm 6.6$ & $-41.7 \pm 3.2$ & \\
\hline AP amplitude ${ }^{b}(\mathrm{mV})$ & $66.5 \pm 3.4$ & $51.7 \pm 8.2$ & $32.2 \pm 8.2$ & $72.3 \pm 3.2$ & \\
\hline AP half-width (ms) & $0.84 \pm 0.06$ & $0.89 \pm 0.08$ & $0.34 \pm 0.05$ & $0.66 \pm 0.01$ & \\
\hline$R_{N}$ Down $(\mathrm{M} \Omega)$ & $29.2 \pm 2.0$ & $25.7 \pm 0.6$ & $53.5 \pm 3.1$ & $22.5 \pm 3.5$ & $52.7 \pm 4.8$ \\
\hline$R_{N}$ Discards $(\mathrm{M} \Omega)$ & $31.6 \pm 2.2$ & $32.4 \pm 3.7$ & $54.0 \pm 0.2$ & $24.5 \pm 6.8$ & $56.3 \pm 4.5$ \\
\hline$R_{N}^{N} \cup p(\mathrm{M} \Omega)$ & $36.6 \pm 2.3$ & $28.2 \pm 1.3$ & $57.3 \pm 3.4$ & $23.6 \pm 4.4$ & $44.6 \pm 2.2$ \\
\hline$\tau_{\text {decay }}$ Down $^{c}(\mathrm{~ms})$ & $9.0 \pm 0.6$ & $8.5 \pm 0.8$ & $2.6 \pm 0.9$ & $8.9 \pm 1.1$ & $20.1 \pm 1.2$ \\
\hline$\tau_{\text {decay }}$ Discards $^{c}(\mathrm{~ms})$ & $10.6 \pm 0.6$ & $6.9 \pm 2.5$ & $2.7 \pm 0.9$ & $9.3 \pm 2.7$ & $21.6 \pm 1.7$ \\
\hline$\tau_{\text {decay }} \mathrm{Up}^{c}(\mathrm{~ms})$ & $12.8 \pm 1.1$ & $16.5 \pm 5.3$ & $2.7 \pm 0.9$ & $9.9 \pm 2.1$ & $16.3 \pm 3.8$ \\
\hline Anomalous rectification, $\mathrm{C}_{\mathrm{AR}}(\mathrm{M} \Omega / \mathrm{nA})$ & $18.7 \pm 3.7(n=10)$ & & & & $0.6 \pm 6.4(n=6)$ \\
\hline$R_{N}$ Down, from $V-I$ curve $(\mathrm{M} \Omega)$ & $27.5 \pm 2.1(n=10)$ & & & & $68.1 \pm 4.8(n=6)$ \\
\hline$R_{N}$ Up, from $V-I$ curve $(\mathrm{M} \Omega)$ & $44.1 \pm 4.0(n=10)$ & & & & \\
\hline
\end{tabular}

All data are given as mean $\pm \mathrm{SE}$.

${ }^{a}$ A total of $60-100 \mu \mathrm{m}$ from the soma.

${ }^{b}$ From threshold.

'Time constant of off-response (starting $1 \mathrm{~ms}$ after end of current pulse).

tion at subthreshold membrane potentials (i.e., increasing $R_{N}$ with depolarization) (Fig. 4A, B).

Unsurprisingly, input resistance dropped dramatically in all neurons when current injections were suprathreshold, causing APs (19.0 $\pm 4.9 \mathrm{M} \Omega ; n=10$ ) (Fig. 4). This drop is attributable to the opening of voltage-gated channels during AP generation (Häusser et al., 2001) and probably explains the difference between our results and previous measurements, because the latter were often from neurons firing APs at high frequencies during Up states $(5-30 \mathrm{~Hz}$ ) (Steriade et al., 1993, 2001; Destexhe and Paré, 1999). 


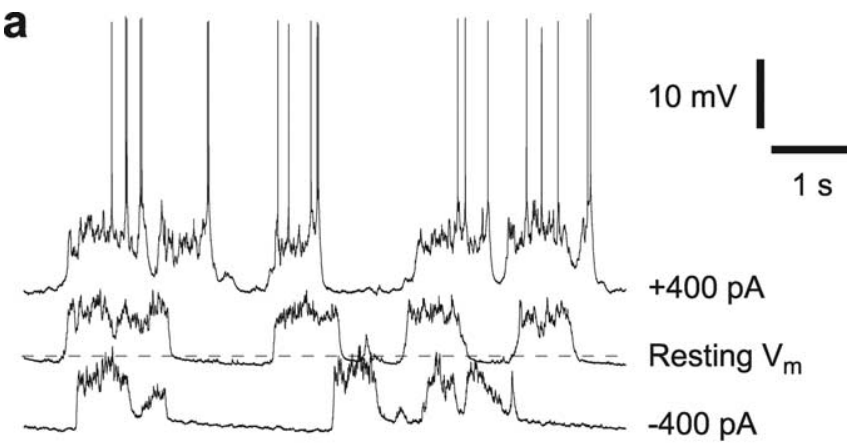

b
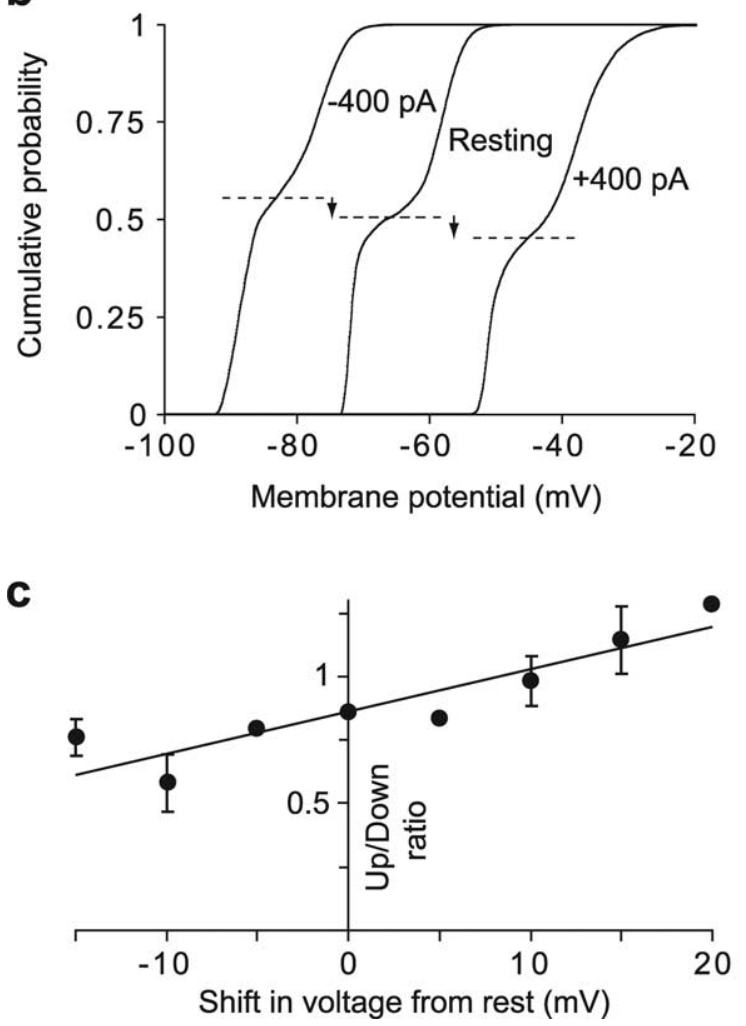

Figure 3. Voltage dependence of Up and Down state durations. $\boldsymbol{a}$, Membrane potential during $\pm 400 \mathrm{pA}$ constant current injection and at rest (no current injection). The dashed line denotes $-80 \mathrm{mV}$. $\boldsymbol{b}$, Cumulative membrane potential histograms corresponding to data shown in $\boldsymbol{a}$. Changes in the position of the inflection point (dashed lines) during current injection are indicated by arrows. c, Effect of constant current injection on the proportion of time spent in Up and Down states. Data points represent means ( \pm SEM). Pooled data from five neurons are shown. The linear fit had a slope of $1.7 \pm 0.9 \%$ per millivolt.

Subthreshold $V-I$ curves were fit assuming a quadratic relationship between injected current and the resulting voltage deflection, $\Delta V=R_{N, 0} \Delta I+c_{\mathrm{AR}} \Delta I^{2}$, where $R_{N, 0}$ denotes input resistance at rest and $c_{\mathrm{AR}}$ is the rectification coefficient (Fig. $4 \mathrm{~B}$ ). Mean values for $R_{N, 0}$ and $c_{\mathrm{AR}}$ were $27.5 \pm 2.1 \mathrm{M} \Omega$ and $18.7 \pm 3.7$ $\mathrm{M} \Omega / \mathrm{nA}$, respectively $\left(n=10 ; c_{\mathrm{AR}}\right.$ range, $\left.4-40 \mathrm{M} \Omega / \mathrm{nA}\right)$. From this description, we derived an analytical function for the voltage dependence of $R_{N}$ (see Materials and Methods), allowing us to calculate the input resistance expected at the Up state membrane potential based on the $V-I$ relationship of the neuron $\left(R_{\mathrm{UpVI}}\right)$. We then compared $R_{\mathrm{Up} V I}$ to $R_{N}$ measured during Up states (Fig. $4 C, D)$. For the 10 neurons analyzed in this way, $R_{\mathrm{Up} V I}$ was $44.1 \pm$ 4.0 $\mathrm{M} \Omega$ and the Up state input resistance was $38.8 \pm 2.7 \mathrm{M} \Omega$. Thus, Up state input resistance was reduced by $12 \%$ compared
A

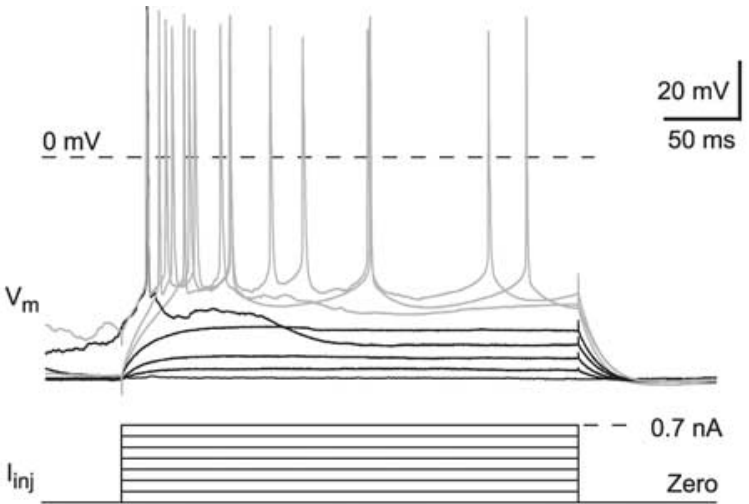

B

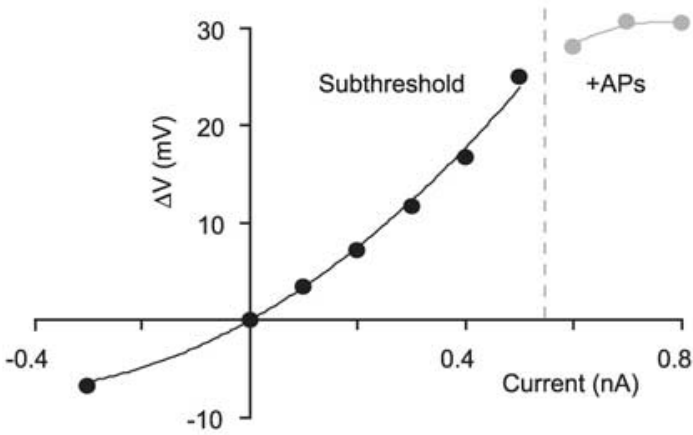

C

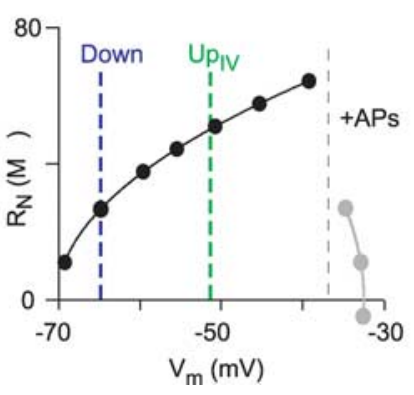

D

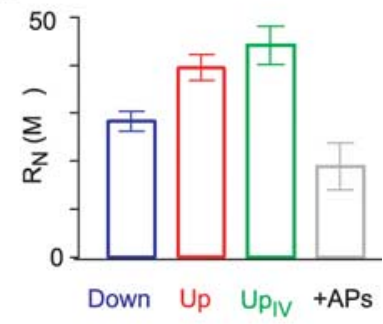

Figure 4. Anomalous rectification overcompensates for synaptic conductance changes. $A$, Voltage recordings from a L2/3 pyramidal neuron during $300 \mathrm{~ms}$ current steps (gray, suprathreshold responses). $\boldsymbol{B}$, Steady-state $V$-/ relationship shows anomalous rectification. Only Down state periods were analyzed. Lines are second-order polynomial fits to subthreshold (black) and suprathreshold (gray) data points. C, Voltage dependence of input resistance (slope of $V$ - I curve in $\boldsymbol{B}$ ). The dashed lines indicate mean Up and Down state membrane potentials for this neuron (green and blue, respectively). Input resistance decreases in the presence of APs. D, Summary of input resistance measurements for 10 neurons under four different conditions: Down (blue) and Up (red) states (measured using brief pulse protocol); intrinsic input resistance at the Up state membrane potential (Up $p_{v i}$ green; estimated from $C$ ) and in the suprathreshold regime (+APs).

with the input resistance without synaptic input at the same membrane potential. By eliminating the effect of membrane potential, this calculation reveals the true effect of synaptic activity on input conductance.

We also eliminated the effect of membrane potential by using constant current injection to depolarize Down states to the Up state membrane potential. This approach revealed an $8 \%$ reduction of $R_{N}$ during Up states (Fig. $\left.5 A, B\right)(35.4 \pm 4.7 \mathrm{M} \Omega$ at $-57.5 \pm 2.6 \mathrm{mV}$ during Up states vs $38.1 \pm 5.1 \mathrm{M} \Omega$ at $-57.6 \pm$ $2.0 \mathrm{mV}$ during "isopotential" Down states; $n=7$ ). Finally, we blocked intrinsic voltage-dependent currents by adding $10 \mathrm{mM}$ QX-314 to the pipette solution. This blocked anomalous rectification in the voltage range of $\mathrm{Up}$ and Down states, linearizing the $V-I$ relationship (Fig. $5 C)\left(R_{N, 0}=68.1 \pm 4.8 \mathrm{M} \Omega ; c_{\mathrm{AR}}=0.6 \pm\right.$ 6.4 , range, $-17-27 \mathrm{M} \Omega / \mathrm{nA} ; c_{\mathrm{AR}}$ significantly less than without 
A

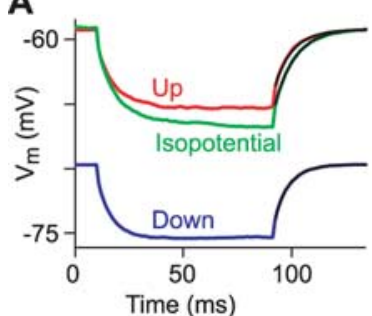

B
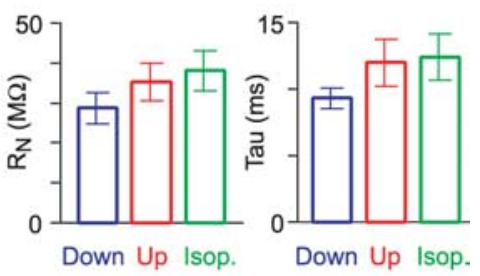

C

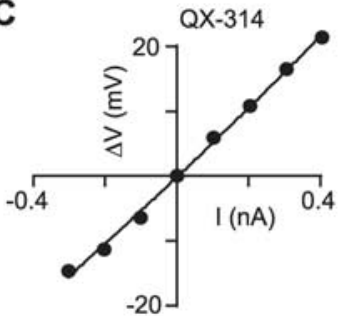

D

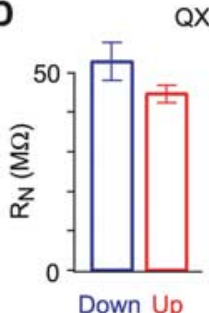

QX-314

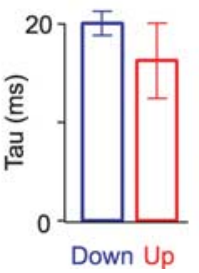

Figure 5. Separation of intrinsic and synaptic conductance changes. $\boldsymbol{A}$, Mean voltage deflections in a $L 2 / 3$ neuron in response to brief current pulses without holding current (blue, Down states; red, Up states) and during depolarizing constant current injection to bring the membrane potential to the Up state level (green trace; isopotential). Single-exponential curves were fit to the voltage decays (black traces). $\boldsymbol{B}$, Summary of input resistance and time constant for experiments with current-induced depolarization to the Up state level $(n=7)$. C, Example $V-I$ relationship with QX-314 in the pipette. The line is a second-order polynomial fit. D, Summary of input resistances and time constants with QX-314 $(n=4)$. Error bars indicate SEM.

QX-314, $p=0.023$, Mann-Whitney test). With QX-314, input resistance was $15 \%$ lower during Up than during Down states (Fig. $5 D)(52.7 \pm 4.8 \mathrm{M} \Omega$ during Down state; $44.6 \pm 2.2 \mathrm{M} \Omega$ during Up state; $n=4)$. We conclude that prominent anomalous rectification in L2/3 neurons explains the increased $R_{N}$ during Up states. If the voltage dependence of $R_{N}$ is accounted for or removed (by comparing isopotential $R_{N}$ values or using QX-314), $R_{N}$ during Up states is $10-15 \%$ lower than during Down states.

\section{Excitatory and inhibitory synaptic conductance changes during Up states}

To quantify the mean synaptic conductance change visible at the soma $\left(g_{\text {syn }}\right)$, we compared somatic input conductance (the reciprocal of $R_{N}$ ) in the absence and presence of synaptic input using the same three approaches used above. Comparison of measured conductances changes (during Up states) with the expected conductance change in the absence of synaptic activity revealed a synaptic conductance change $g_{\text {syn }}$ of $8.2 \pm 2.0 \mathrm{nS}$ (range, $0-17 \mathrm{nS}$; $n=10)$. Calculating $g_{\text {syn }}$ from the difference of conductances in Up states and isopotential Down states and from the conductance difference with anomalous rectification blocked by QX-314 yielded comparable values of $2.2 \pm 1.4(n=7)$ and $3.0 \pm 1.0 \mathrm{nS}$ $(n=4)$, respectively. Thus, only a small conductance change in the range of 2-10 nS is visible at the soma during cortical Up states.

We used this conductance change to estimate the number of active synapses and presynaptic firing rates by reconstructing cortical Up states in numerical simulations. We started with a point conductance model, in which resting membrane properties and anomalous rectification were matched to experimental results (Fig. 6A). Excitatory and inhibitory synaptic inputs were modeled as Poisson-distributed presynaptic spike trains evoking AMPA- and $\mathrm{GABA}_{\mathrm{A}}$-type postsynaptic currents, respectively (see Materials and Methods). Assuming a total conductance change of $8 \mathrm{nS}$ we first explored the consequences of changing the mean inhibition/excitation ratio $\left(\left\langle g_{\mathrm{i}}\right\rangle /\left\langle g_{\mathrm{e}}\right\rangle\right)$. The model reproduced Up states with mean depolarizations and input resistance increases consistent with our experimental results if $\left\langle g_{\mathrm{i}}\right\rangle /\left\langle g_{\mathrm{e}}\right\rangle$ was $<0.3$ (Fig. $6 B, C)$. A ratio of 0.1 gave the closest match, resulting in $15 \mathrm{mV}$ mean depolarization and $12.5 \%$ net input resistance increase during Up states (Fig. 6B, $C$ ).

In contrast, if we excluded anomalous rectification from our model and assumed a large decrease in input resistance during $\mathrm{Up}$ states, a $\left\langle g_{\mathrm{i}}\right\rangle /\left\langle g_{\mathrm{e}}\right\rangle$ ratio of $\sim 3$ was required to produce $15 \mathrm{mV}$ Up states (supplemental Fig. 2, available at www.jneurosci.org as supplemental material). This value is comparable with previous estimates, which have suggested that inhibition dominates excitation (Destexhe et al., 2003).

The ratio of inhibition to excitation also determines the reversal potential of Up states (Wilson and Kawaguchi, 1996; Shu et al., 2003). Under our conditions, a ratio of 0.1 would produce an Up state reversal potential of $-9 \mathrm{mV}$ (see Materials and Methods). We directly measured the reversal potential of Up states in vivo by depolarizing QX-314-filled neurons to different membrane potentials and measuring the mean voltage difference between Up and Down states. Up states were defined using a simultaneously measured electrocorticogram (Waters and Helmchen, 2004). The mean reversal potential was $-10.4 \mathrm{mV}$ (Fig. $6 D)(n=5$ neurons), which corresponds to a mean conductance ratio $\left(\left\langle g_{\mathrm{i}}\right\rangle /\right.$ $\left.\left\langle g_{\mathrm{e}}\right\rangle\right)$ of 0.12 . Thus, both simulations and experiments suggest that inhibition contributes $\sim 10 \%$ to the total conductance change.

\section{Estimate of numbers of activated synapses from a compartmental model}

Next, we used a compartmental model of a morphologically reconstructed L2/3 pyramidal neuron to convert the conductance change into an estimate of the number of active synapses (Fig. $7 A, B)$. The neuron was equipped with anomalous rectification so that it reproduced the experimentally measured $V-I$ curves. A total of 10,000 excitatory and 2000 inhibitory synapses was uniformly distributed throughout the dendritic tree (DeFelipe and Farinas, 1992). As in the point conductance model, Up states with a mean depolarization of $15 \mathrm{mV}$ and increased somatic input resistance were generated with low rates of synaptic input $(0.33$ and $0.067 \mathrm{~Hz}$ per input for excitatory and inhibitory synapses, respectively). This corresponds to $\sim 3$ excitatory and 1.3 inhibitory synapses activated per millisecond.

Interestingly, our simple model captured several experimentally observed features of L2/3 neurons. First, although voltage fluctuations during Up states increased with distance from the soma, the mean depolarization was nearly constant throughout the apical dendritic tree (Fig. 7C), similar to our in vivo whole-cell recordings from soma, apical dendrite, and distal tuft branches (Waters and Helmchen, 2004). Second, several studies reported that sensory-evoked EPSPs show reduced amplitudes during Up states (Petersen et al., 2003; Sachdev et al., 2004). Simulating this situation in our model (see Materials and Methods), we found that the compound EPSP amplitude during Up states was reduced to $86 \%$ of the Down state amplitude despite a mean input resistance increase of $27 \%$ (Fig. $7 D$ ). In our model, this attenuation of the compound EPSP is caused exclusively by the reduction in driving force. The larger attenuation observed for sensoryevoked responses (Petersen et al., 2003; Sachdev et al., 2004) presumably results from additional factors such as synaptic depression and feedforward and feedback inhibition.

Finally, we used our compartmental model to estimate how many synapses are required to depolarize the neuron to different membrane potentials. We considered two situations: asynchro- 
nous, distributed background activity and synchronous synaptic inputs onto the basal arbors (such as occurs during activation of a specific afferent pathway). The excitation/inhibition ratio was kept constant at 0.1 . With asynchronous synaptic input and without anomalous rectification, the mean depolarization per synapse decreased as the frequency of inputs was increased (Fig. 8A). This decrease is attributable to the diminished driving force for excitatory currents at depolarized membrane potentials. In the presence of anomalous rectification, synaptic inputs summated more effectively but a small decrease in mean depolarization per synapse persisted despite the increase in somatic input resistance. Thus, anomalous rectification did not fully linearize the relationship between net depolarization and frequency of synaptic inputs. Nevertheless, only $\sim 14$ active synapses per millisecond were required to reach AP threshold. In the case of synchronous excitatory inputs to the basal dendrites, more than one hundred synapses were required to bring the neuron to AP threshold during Up states (Fig. $8 \mathrm{~B}$ ). We conclude that, during background network activity, spiking is typically driven by tens to little more than a hundred synapses, depending on their rate of activation and their synchrony.

\section{Discussion}

We have shown that the input resistance of L2/3 neurons increases during cortical Up states and that this is because anomalous rectification dominates the synaptic conductance increase. We conclude that, first, Up states are not high-conductance states in L2/3 pyramidal neurons but are associated with stable or reduced input conductance. Second, anomalous rectification balances the synaptic conductance increase in vivo, thereby reducing the shunting problem. Finally, few synapses are active during Up states $(<5$ per millisecond) and the majority ( $\sim 90 \%$ ) are excitatory.

\section{Input resistance during Up states}

Using three complementary methods, we found a 10-30\% mean increase in input resistance during Up states, equivalent to a $10-$ $25 \%$ drop in input conductance. This resembles in vivo results from neostriatal spiny projection neurons (Wilson and Groves, 1981; Wilson and Kawaguchi, 1996) but contrasts with previous studies of cortical neurons that reported twofold to fivefold decreases in input resistance during Up states (Paré et al., 1998; Steriade et al., 2001; Léger et al., 2005) (for review, see Destexhe et al., 2003) (but see Cowan and Wilson, 1994). This discrepancy is not related to the recording technique, because we obtained similar results with whole-cell patch pipettes and sharp microelectrodes. Age and species differences are also unlikely to be responsible, because the conductance change necessary to produce a $50-80 \%$ decrease in input resistance would be approximately an order of magnitude larger than we measured here [compare Fig. 6 and supplemental Fig. 2 (available at www.jneurosci.org as supplemental material)], and it seems unlikely that total synaptic
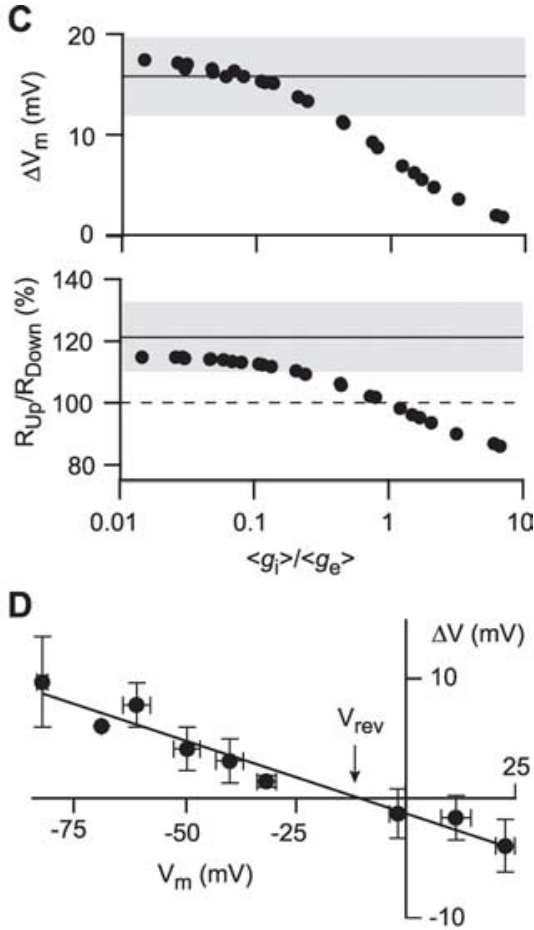

Time (s)

Figure 6. Numerical reconstruction of Up states indicates a low inhibition/excitation ratio. $\boldsymbol{A}, \mathrm{A}$ point conductance model with 作 measurement. The membrane potential difference between Up and Down states $(\Delta V)$ is plotted against mean Down state membrane potential (controlled by current injection). Data are pooled in $10 \mathrm{mV}$ bins from five neurons. The regression line reverses sign at $-10.4 \mathrm{mV}$. Error bars indicate SEM.

input would differ sufficiently between species or between young adult and adult animals.

The most likely explanation is that previously reported decreases in input resistance resulted principally from the opening of voltagegated channels during APs, not from synaptic conductances. Consistent with this explanation, AP firing caused an apparent input resistance decrease of $50 \%$ or more in our recordings (Fig. 4). In agreement with recent in vivo studies (Margrie et al., 2002; Brecht et al., 2003), we observed few APs during Up states. In contrast, other authors have reported high-frequency (tens of hertz) firing during Up states (Steriade et al., 2001). Why different authors report such disparate firing rates during Up states is unclear. Leak around the pipette may be a contributory factor in sharp microelectrode penetrations, although in our microelectrode recordings firing rates were similar to whole-cell recordings $(\sim 0.1 \mathrm{~Hz})$. Low firing rates have been reported in cell-attached recordings in vivo (Margrie et al., 2002), demonstrating that dialysis with patch pipettes is not a critical factor. Furthermore, neocortical AP activity is sparse in unanesthetized animals (Margrie et al., 2002; Petersen et al., 2003). Hence input resistances may be determined principally by subthreshold intrinsic currents and synaptic activity in awake animals.

\section{Anomalous rectification}

Anomalous rectification occurs in many cell types in the CNS, including spinal cord motoneurons (Nelson and Frank, 1967), hippocampal pyramidal (Hotson et al., 1979), cerebellar Purkinje 


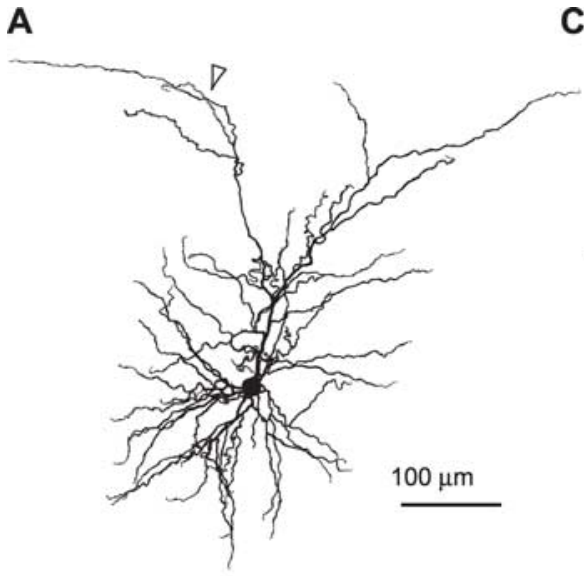

C
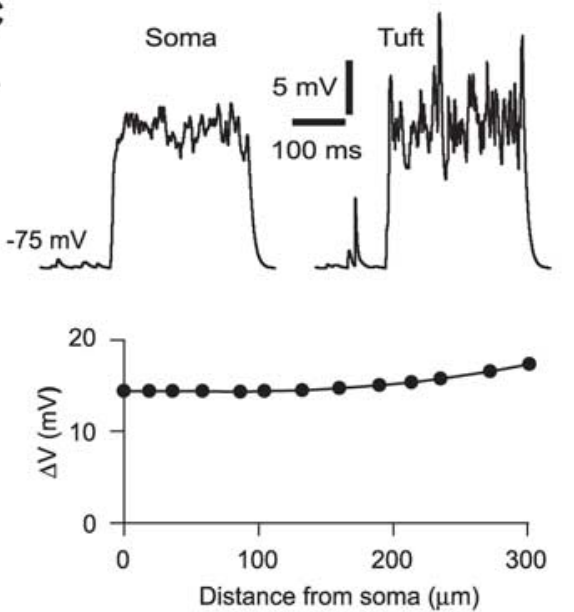

B

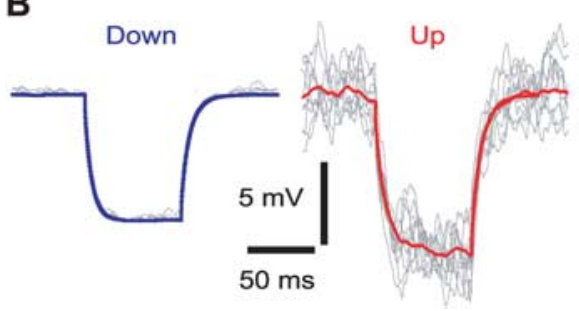

D

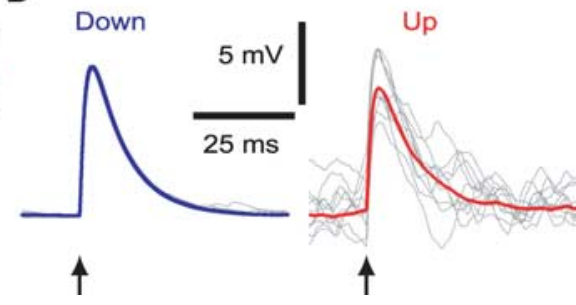

Figure 7. Full morphology model of Up states. A, A model from a morphologically reconstructed L2/3 pyramidal neuron was equipped with anomalous rectification and with 10,000 excitatory and 2000 inhibitory, uniformly distributed synaptic inputs. Presynaptic mean spike rates were $0.33 \mathrm{~Hz}$ for excitatory and $0.067 \mathrm{~Hz}$ for inhibitory synapses during Up states and 100 -fold lower during Down states $\left(\left\langle g_{i}\right\rangle /\left\langle g_{e}\right\rangle=0.1\right)$. B, Input resistance (measured with current pulses) was $27 \%$ higher during Up states. Gray, Ten individual traces; red and blue, mean for 80 trials in Up and Down states, respectively. Initial membrane potentials were -75 and $-60.8 \mathrm{mV}$ for Down and Up states. $C$, Examples of simulated Up state voltage traces in soma and a dendritic tuft branch ( $\boldsymbol{A}$, arrowhead). Below, Mean Up state amplitude as a function of distance from the soma. $\boldsymbol{D}$, Reduced EPSP amplitude during Up states. Compound EPSPs of $\sim 10 \mathrm{mV}$ amplitude were generated by synchronous activation (arrow) of 25 excitatory synapses on the basal dendrites (mean distance, $68 \mu \mathrm{m}$ ). Gray, Ten individual traces; red and blue, averages of 80 traces for Up and Down states, respectively. Initial membrane potentials were -75 and $-61.1 \mathrm{mV}$ for Down and Up states.
A

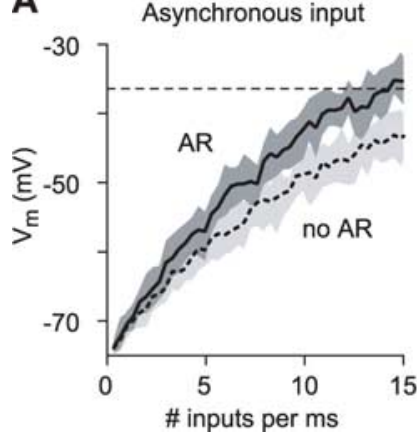

B

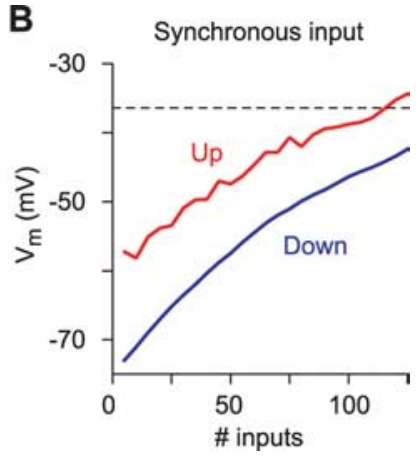

Figure 8. Depolarization per active synapse in the full morphology model. $\boldsymbol{A}$, Steady-state depolarization with asynchronous (Poisson-distributed), spatially distributed inputs as a function of number of active excitatory synapses per millisecond in the presence and absence of anomalous rectification (AR). The lines and gray shaded areas represent mean and minimum/ maximum values, respectively. The dashed horizontal line is AP threshold of $-36.4 \mathrm{mV}$ (Table 1). An exponential fit to the relationship with anomalous rectification (data not shown) crossed AP threshold at 14.2 synapses per millisecond. $\boldsymbol{B}$, Peak EPSP amplitudes of synchronous excitatory inputs to the basal dendrites from Up or Down states as a function of the number of inputs.

(Llinás and Sugimori, 1980), locus ceruleus (Osmanovic and Shefner, 1987), neostriatal spiny (Wilson, 1992), and cortical pyramidal neurons (Connors et al., 1982; Stafstrom et al., 1982; Sutor and Zieglgänsberger, 1987; Cowan and Wilson, 1994). Hence, anomalous rectification counterbalancing synaptic con- ductance changes is likely to be widespread in the CNS. Although anomalously rectifying currents have been identified as a key factor influencing Up states in neostriatal spiny neurons (Wilson 1992) and despite descriptions of anomalous rectification in the cortical literature, the role of anomalous rectification during Up states in cortical pyramidal neurons has been mostly overlooked.

Several voltage-dependent ion channels may contribute to anomalous rectification. Inwardly rectifying $\mathrm{K}^{+}$currents are typically responsible at hyperpolarized voltages (Osmanovic and Shefner, 1987; Sutor and Zieglgänsberger, 1987; Nisenbaum and Wilson, 1995). In the depolarizing direction, inward sodium and calcium currents have also been implicated (Stafstrom et al., 1985; Sutor and Zieglgänsberger, 1987). Here, we have shown that QX-314 blocked anomalous rectification in the voltage range of Up and Down states, consistent with previous studies of cortical pyramidal neurons (Stafstrom et al., 1982, 1985; Sutor and Zieglgänsberger, 1987).

\section{Synaptic conductance change during Up states}

During Up states, the mean synaptic conductance change visible at the soma is $8 \mathrm{nS}$. This is $>10$-fold smaller than estimates from several in vivo studies (Destexhe et al., 2003), but comparable with recent in vitro and in vivo estimates from ferret cortex (4-32 nS) (McCormick et al., 2003; Shu et al., 2003; Haider et al., 2006). In contrast to these ferret studies, which suggest balanced inhibition and excitation, our simulations and reversal potential measurements point to a lower inhibition/excitation ratio of $\sim 0.1$, indicating that inhibition is sparse during cortical Up states. Reversal potential measurements are, however, prone to errors, including junction potentials and misestimation of the reversal potential of IPSPs $\left(E_{\mathrm{i}}\right)$. In 3- to 5-week-old rats, $E_{\mathrm{i}}$ has been measured at around $-70 \mathrm{mV}$ (Luhmann and Prince, 1991; Gulledge and Stuart, 2003). To estimate the possible error in our calculations, we recalculated $\left\langle g_{\mathrm{i}}\right\rangle /$ $\left\langle g_{\mathrm{e}}\right\rangle$ with $E_{\mathrm{i}}=-70 \mathrm{mV}$ (instead of $-95 \mathrm{mV}$ ) and assuming a 10 $\mathrm{mV}$ junction potential, yielding $\left\langle g_{\mathrm{i}}\right\rangle /\left\langle g_{\mathrm{e}}\right\rangle=0.41$, approximately fourfold higher than our estimate of $\left\langle g_{\mathrm{i}}\right\rangle /\left\langle g_{\mathrm{e}}\right\rangle=0.1$. Our conclusion, that the majority of synaptic drive during Up states is excitatory, not inhibitory, is therefore robust even against sizable possible errors in our calculations.

Our simulations indicate that postsynaptic activity is sparse during Up states ( $\sim 0.3 \mathrm{~Hz}$ for excitatory synapses). To convert this number into average firing rates of presynaptic neurons, one needs to divide it by the presynaptic release probability, which is unknown for many cortical connections. Assuming a release probability of 0.8 , as estimated for connections between L5 pyramidal neurons and from L4 spiny stellates to L2/3 pyramidal neurons (Markram et al., 1997; Silver et al., 2003), would give a presynaptic firing rate of $\sim 0.4 \mathrm{~Hz}$ during Up states. Neurons spent approximately equal amounts of time in Up and in Down 
states (Table 1), so presynaptic firing rates averaged over time (including both states) would be $0.2 \mathrm{~Hz}$. This is consistent with the low spontaneous firing rates observed in our whole-cell recordings and in previous studies (Margrie et al., 2002; Brecht et al., 2003; Kerr et al., 2005).

Our recordings were obtained from somata, far from most active synapses. Presumably, conductance changes in some locations in the dendrites will be greater. Distance-dependent attenuation of conductance along dendrites is severe (Koch et al., 1990; Williams, 2004). Hence, a drop in input resistance might occur locally in a dendrite, causing local shunting but little effect at the soma (Rall, 1967; Koch et al., 1990). In addition, activation of different combinations of synapses during different Up states (either close to the soma or more distally) might produce different conductance changes at the soma. This could explain the variability that we observed in the amplitudes of voltage deflections between individual Up states (Fig. $1 B$ ).

Our simple model reproduced the experimental results assuming a uniform distribution of synaptic input throughout the dendrites. This assumption is probably reasonable for excitatory but not for inhibitory synapses (DeFelipe and Fariñas, 1992; Markram et al., 2004; Waters and Helmchen, 2004). Future models might consider the effect of the location of inhibitory synapses in the dendritic tree. Furthermore, our model needs to be extended to the suprathreshold regime, because local dendritic spikes generated by synchronous, clustered inputs (Gasparini and Magee, 2006) might play an important role in synaptic integration.

\section{Functional implications}

Sparse background activity in cortical networks may enable the cortex to use computationally and energetically efficient sparse coding schemes despite ongoing network activity (Olshausen and Field, 2004). In particular, the influence of synaptic background on single-cell computation may be less than previously thought. With few active synapses and anomalously rectifying currents, dendrites become electronically more compact during synaptic background activity (the opposite is expected for a linear membrane) (Koch, 1999). This may explain why AP backpropagation is conserved in vivo, even during synaptic activity (Buzsáki and Kandel, 1998; Charpak et al., 2001; Quirk et al., 2001; Waters and Helmchen, 2004; Waters et al., 2005). Likewise, both forward propagation of dendritically initiated spikes (Golding and Spruston, 1998; Larkum et al., 2001) and amplification of EPSPs by voltage-gated channels (Stuart and Sakmann, 1995) should be preserved during network activity in vivo.

Interestingly, in L5 pyramidal neurons, anomalous rectification becomes stronger during postnatal development, compensating for the decrease in input resistance that occurs in parallel (Kasper et al., 1994). Thus, anomalous rectification could tune network activity during development. Furthermore, neuromodulatory regulation of voltage-dependent currents operating in the subthreshold range could affect the emergence of different network states.

In summary, we have shown that surprisingly few synapses drive spontaneous depolarizations in L2/3 pyramidal neurons in the anesthetized rat. Their contribution to membrane conductance is opposed by anomalous rectification, which enhances the effectiveness of each synapse during volleys of synaptic activity and decreases the influence of synaptic activity on dendritic properties. Against such a low synaptic background, a few precisely timed synaptic inputs (on the order of 100) should be suf- ficient to produce and maintain specific activation patterns in cortical circuits.

\section{References}

Ali AB, Rossier J, Staiger JF, Audinat E (2001) Kainate receptors regulate unitary IPSCs elicited in pyramidal cells by fast-spiking interneurons in the neocortex. J Neurosci 21:2992-2999.

Bernander O, Douglas RJ, Martin KA, Koch C (1991) Synaptic background activity influences spatiotemporal integration in single pyramidal cells. Proc Natl Acad Sci USA 88:11569-11573.

Brecht M, Roth A, Sakmann B (2003) Dynamic receptive fields of reconstructed pyramidal cells in layers 3 and 2 of rat somatosensory barrel cortex. J Physiol (Lond) 553:243-265.

Buzsáki G, Kandel A (1998) Somadendritic backpropagation of action potentials in cortical pyramidal cells of the awake rat. J Neurophysiol 79:1587-1591.

Charpak S, Mertz J, Beaurepaire E, Moreaux L, Delaney K (2001) Odorevoked calcium signals in dendrites of rat mitral cells. Proc Natl Acad Sci USA 98:1230-1234.

Connors BW, Gutnick MJ, Prince DA (1982) Electrophysiological properties of neocortical neurons in vitro. J Neurophysiol 48:1302-1320.

Cowan RL, Wilson CJ (1994) Spontaneous firing patterns and axonal projections of single corticostriatal neurons in the rat medial agranular cortex. J Neurophysiol 71:17-32.

DeFelipe J, Fariñas I (1992) The pyramidal neuron of the cerebral cortex: morphological and chemical characteristics of the synaptic input. Prog Neurobiol 39:563-607.

Destexhe A, Paré D (1999) Impact of network activity on the integrative properties of neocortical pyramidal neurons in vivo. J Neurophysiol 81:1531-1547.

Destexhe A, Rudolph M, Paré D (2003) The high-conductance state of neocortical neurons in vivo. Nat Rev Neurosci 4:1-14.

Feldmeyer D, Lübke J, Silver RA, Sakmann B (2002) Synaptic connections between layer 4 spiny neurone-layer $2 / 3$ pyramidal cell pairs in juvenile rat barrel cortex: physiology and anatomy of interlaminar signalling within a cortical column. J Physiol (Lond) 538:803-822.

Gasparini S, Magee JC (2006) State-dependent dendritic computation in hippocampal CA1 pyramidal neurons. J Neurosci 26:2088-2100.

Golding NL, Spruston N (1998) Dendritic sodium spikes are variable triggers of axonal action potentials in hippocampal CA1 pyramidal neurons. Neuron 21:1189-1200.

Gulledge AT, Stuart GJ (2003) Excitatory actions of GABA in the cortex. Neuron 37:299-309.

Haider B, Duque A, Hasenstaub AR, McCormick DA (2006) Neocortical network activity in vivo is generated through a dynamic balance of excitation and inhibition. J Neurosci 26:4535-4545.

Häusser M, Roth A (1997) Estimating the time course of the excitatory synaptic conductance in neocortical pyramidal cells using a novel voltage jump method. J Neurosci 17:7606-7625.

Häusser M, Spruston N, Stuart GJ (2000) Diversity and dynamics of dendritic signaling. Science 290:739-744.

Häusser M, Major G, Stuart GJ (2001) Differential shunting of EPSPs by action potentials. Science 291:138-141.

Hines ML, Carnevale NT (1997) The NEURON simulation environment. Neural Comput 9:1179-1209.

Hotson JR, Prince DA, Schwartzkroin PA (1979) Anomalous rectification in hippocampal neurons. J Neurophysiol 42:889-895.

Kasper EM, Larkman AU, Lübke J, Blakemore C (1994) Pyramidal neurons in layer 5 of the rat visual cortex. II. Development of electrophysiological properties. J Comp Neurol 339:475-494.

Katz B (1949) Les constants electriques de la membrane du muscle. Arch Sci Physiol 3:285-299.

Kerr JN, Greenberg D, Helmchen F (2005) Imaging input and output of neocortical networks in vivo. Proc Natl Acad Sci USA 102:14063-14068.

Koch C (1999) Biophysics of computation: information processing in single neurons. New York: Oxford UP.

Koch C, Douglas R, Wehmeier U (1990) Visibility of synaptically induced conductance changes: theory and simulations of anatomically characterized cortical pyramidal cells. J Neurosci 12:1728-1744.

Larkum ME, Zhu JJ, Sakmann B (2001) Dendritic mechanisms underlying the coupling of the dendritic with the axonal action potential initiation 
zone of adult rat layer 5 pyramidal neurons. J Physiol (Lond) 533:447-466.

Léger J-F, Stern AS, Aertsen A, Heck D (2005) Synaptic integration in rat prefrontal cortex shaped by network activity. J Neurophysiol 93:281-293.

Llinás R, Sugimori M (1980) Electrophysiological properties of in vitro Purkinje cell somata in mammalian cerebellar slices. J Physiol (Lond) 305:171-195.

Lübke J, Roth A, Feldmeyer D, Sakmann B (2003) Morphometric analysis of the columnar innervation domain of neurons connecting layer 4 and layer 2/3 of juvenile rat barrel cortex. Cereb Cortex 13:1051-1063.

Luhmann HJ, Prince DA (1991) Postnatal maturation of the GABAergic system in rat neocortex. J Neurophysiol 65:247-263.

Magee JC (2000) Dendritic integration of excitatory synaptic input. Nat Rev Neurosci 1:181-190.

Margrie TW, Brecht M, Sakmann B (2002) In vivo, low-resistance, wholecell recordings from neurons in the anaesthetized and awake mammalian brain. Pflügers Arch 444:491-498.

Markram H, Lubke J, Frotscher M, Roth A, Sakmann B (1997) Physiology and anatomy of synaptic connections between thick tufted pyramidal neurones in the developing rat neocortex. J Physiol (Lond) 500:409-440.

Markram H, Toledo-Rodriguez M, Gupta A, Silberberg G, Wu C (2004) Interneurons of the neocortical inhibitory system. Nat Rev Neurosci 5:793-807.

McCormick DA, Shu Y, Hasenstaub A, Sanchez-Vivez M, Badoual M, Bal T (2003) Persistent cortical activity: mechanisms of generation and effects on neuronal excitability. Cereb Cortex 13:1219-1231.

Nelson PG, Frank K (1967) Anomalous rectification in cat spinal motoneurons and effect of polarizing currents on excitatory postsynaptic potential. J Neurophysiol 30:1097-1113.

Nisenbaum ES, Wilson CJ (1995) Potassium currents responsible for inward and outward rectification in rat neostriatal spiny projection neurons. J Neurosci 15:4449-4463.

Olshausen BA, Field DJ (2004) Sparse coding of sensory inputs. Curr Opin Neurobiol 14:481-487.

Osmanovic SS, Shefner SA (1987) Anomalous rectification in rat locus coeruleus neurons. Brain Res 417:161-166.

Paré D, Shink E, Gaudreau H, Destexhe A, Lang EJ (1998) Impact of spontaneous synaptic activity on the resting properties of cat neocortical pyramidal neurons in vivo. J Neurophysiol 79:1450-1460.

Petersen CCH, Hahn TTG, Mehta M, Grinvald A, Sakmann B (2003) Interaction of sensory responses with spontaneous depolarization in layer $2 / 3$ barrel cortex. Proc Natl Acad Sci USA 100:13638-13643.

Polsky A, Mel BW, Schiller J (2004) Computational subunits in thin dendrites of pyramidal cells. Nat Neurosci 7:621-627.

Quirk M, Blum KI, Wilson MA (2001) Experience-dependent changes in extracellular spike amplitude may reflect regulation of dendritic action potential back-propagation in rat hippocampal pyramidal cells. J Neurosci 21:240-248.

Rall W (1967) Distinguishing theoretical synaptic potentials computed for different soma-dendritic distributions of synaptic input. J Neurophysiol 30:1138-1168.
Rall W (1969) Time constants and electrotonic length of membrane cylinders and neurons. Biophys J 9:1483-1508.

Sachdev RN, Ebner FF, Wilson CJ (2004) The effect of sub-threshold up and down states on the whisker evoked response in somatosensory cortex. J Neurophysiol 92:3511-3521.

Shu Y, Hasenstaub A, McCormick DA (2003) Turning on and off recurrent balanced cortical activity. Nature 423:288-293.

Silver RA, Lubke J, Sakmann B, Feldmeyer D (2003) High-probability uniquantal transmission at excitatory synapses in barrel cortex. Science 302:1981-1984.

Spain WJ, Schwindt PC, Crill W (1987) Anomalous rectification in neurons from cat sensorimotor cortex in vitro. J Neurophysiol 57:1555-1576.

Stafstrom CE, Schwindt PC, Crill EC (1982) Negative slope conductance due to a persistent subthreshold sodium current in cat neocortical neurons in vitro. Brain Res 236:221-226.

Stafstrom CE, Schwindt PC, Chubb MC, Crill EC (1985) Properties of persistent sodium conductance and calcium conductance of layer $\mathrm{V}$ neurons from cat sensorimotor cortex in vitro. J Neurophysiol 53:153-169.

Steriade M (2001) Impact of network activities on neuronal properties in corticothalamic systems. J Neurophysiol 86:1-39.

Steriade M, Nuñez A, Amzica F (1993) A novel slow ( $<1 \mathrm{~Hz}$ ) oscillation of neocortical neurons in vivo: depolarizing and hyperpolarizing components. J Neurosci 73:3252-3285.

Steriade M, Timofeev I, Grenier F (2001) Natural waking and sleep states: a view from inside neocortical neurons. J Neurophysiol 85:1969-1985.

Stuart G, Sakmann B (1995) Amplification of EPSPs by axosomatic sodium channels in neocortical pyramidal neurons. Neuron 15:1065-1076.

Sutor B, Zieglgänsberger W (1987) A low-voltage activated, transient calcium current is responsible for the time-dependent depolarizing inward rectification of rat neocortical neurons in vitro. Pflügers Arch 410:102-111.

Waters J, Helmchen F (2004) Boosting of action potential backpropagation by neocortical network activity in vivo. J Neurosci 24:11127-11136.

Waters J, Larkum M, Sakmann B, Helmchen F (2003) Supralinear $\mathrm{Ca}^{2+}$ influx into dendritic tufts of layer $2 / 3$ neocortical pyramidal neurons in vitro and in vivo. J Neurosci 23:8554-8567.

Waters J, Schaefer A, Sakmann B (2005) Backpropagating action potentials in neurones: measurement, mechanisms and potential functions. Prog Biophys Mol Biol 87:145-170.

Williams SR (2004) Spatial compartmentalization and functional impact of conductance in pyramidal neurons. Nat Neurosci 7:961-967.

Wilson CJ (1992) Dendritic morphology, inward rectification, and the functional properties of neostriatal neurons. In: Single neuron computation (McKenna T, Davis J, Zornetzer SF, eds), pp. 141-172. San Diego: Academic.

Wilson CJ, Groves PM (1981) Spontaneous firing patterns of identified spiny neurons in the rat neostriatum. Brain Res 220:67-80.

Wilson CJ, Kawaguchi Y (1996) The origins of two-state spontaneous membrane potential fluctuations of neostriatal spiny neurons. J Neurosci 16: 2397-2410. 